\title{
Revelando desfechos do cuidado com a mulher em situação de violência conjugal
}

Revealing outcomes of care for women in situations of domestic violence Revelación de resultados del cuidado de mujeres en situación de violencia conyugal

Jordana Brock Carneiro ${ }^{1}$ id https://orcid.org/0000-0002-7496-852X

Nadirlene Pereira Gomes ${ }^{1}$ (i) https://orcid.org/0000-0002-6043-3997

Lilian Conceição Guimarães de Almeida ${ }^{1}$ ic https://orcid.org/0000-0001-6940-9187,

Luana Moura Campos ${ }^{1}$ iD https://orcid.org/0000-0001-5671-1977

Júlia Renata Fernandes Magalhães ${ }^{1}$ ic https://orcid.org/0000-0003-0631-2374 Josinete Gonçalves dos Santos Lírio ${ }^{1}$ ib https://orcid.org/0000-0001-7610-3186

Ionara Rocha das Virgens ${ }^{1}$ ID https://orcid.org/0000-0002-6906-514X Dália Sousa Gonçalves da Costa ${ }^{2}$ id hitps://orcid.org/0000-0001-5184-3487

Como citar:

Carneiro JB, Gomes NP, Almeida LC, Campos $L M$, Magalhães JR, Lírio JG, et al. Revelando desfechos do cuidado com a mulher em situação de violência conjugal. Acta Paul Enferm. 2021;34:eAPE001555.

DOI http://dx.doi.org/10.37689/actaape/2021A0001555

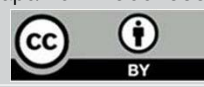

Descritores

Violência contra a mulher; Violência por parceiro intimo; Saúde da mulher; Adaptação psicológica; Atenção primária à saúde

Keywords

Violence against women; Intimate partner violence; Women's health; Adaptation, psychological; Primary health care

Descriptores Violencia contra la mujer; Violencia de pareja: Salud de la mujer; Adaptación psicológica; Atención primaria de salud

Submetido 21 de Junho de 2020 Aceito 1 de Março de 2021

Autor correspondente Jordana Brock Carneiro

E-mail: jordanabrock@yahoo.com.br

\section{Resumo}

Objetivo: Revelar as percepções dos profissionais de saúde sobre os desfechos do cuidado com a mulher em situação de violência conjugal.

Métodos: Estudo de abordagem qualitativa embasado no referencial teórico-metodológico da Teoria Fundamentada nos Dados. Para a coleta de dados, foram realizadas entrevistas com dois grupos amostrais compostos, no total, de 31 profissionais de saúde de nível superior integrantes da equipe mínima ou do Núcleo de Apoio à Saúde da Família de Unidades de Saúde da Família de um distrito sanitário localizado em uma capital do Nordeste brasileiro.

Resultados: A partir das narrativas das participantes da pesquisa, foram elucidados desfechos do cuidado com a mulher em situação de violência conjugal, os quais estão organizados em duas categorias representativas: Propiciando o enfrentamento da violência conjugal a partir do fortalecimento da mulher e Comprometendo o enfrentamento da violência conjugal em razão do não cuidado, sendo essa última composta por duas subcategorias intituladas Vulnerabilizando a mulher para permanência na relação abusiva e Suscetibilizando a mulher para 0 adoecimento/morte.

Conclusão: A oferta de uma assistência qualificada nos serviços impacta diretamente na vida das mulheres que vivenciam violência, seja positiva ou negativamente, uma vez que o não cuidado pode reverberar em agravamentos à saúde da mulher e resultar, inclusive, em seu óbito, enquanto que, ao prestar uma assistência acolhedora, o profissional favorece o empoderamento feminino e, assim, contribui para o enfrentamento da violência conjugal.

\section{Abstract}

Objective: To reveal healthcare professionals' perceptions about the outcomes of care for women in situations of marital violence.

Methods: This is a qualitative approach study based on the Grounded Theory theoretical-methodological framework. For data collection, interviews were carried out with two sample groups composed, in total, of 31 higher education healthcare professionals, members of the minimum team or of Family Health Support Nucleus of Family Health Units in a health district in a capital of northeastern Brazil.

Results: From the participants' narratives, outcomes of care for women in situations of marital violence were elucidated, which are organized into two representative categories: Providing marital violence tackling from women empowerment and Compromising marital violence tackling due to non-care, the latter being composed of two subcategories entitled Vulnerating women to remain in an abusive relationship and Making women susceptible to illness/death. 
Conclusion: The offer of qualified assistance in services directly impacts the lives of women who experience violence, whether positively or negatively, since non-care can reverberate in women's health and even result in their death. Providing a welcoming assistance, professionals favor female empowerment, thus contributing to the tackling conjugal violence.

\section{Resumen}

Objetivo: Revelar las percepciones de los profesionales de la salud sobre los resultados del cuidado de mujeres en situación de violencia conyugal.

Métodos: Estudio de enfoque cualitativo basado en el marco referencial teórico-metodológico de la teoría fundamentada en los datos. Para la recopilación de datos se realizaron encuestas con dos grupos de muestras compuestos, en total, por 31 profesionales de la salud de nivel superior integrantes del equipo mínimo o del Núcleo de Apoyo a la Salud de la Familia de Unidades de Salud de la Familia de un distrito sanitario ubicado en una capital de la región Nordeste brasileña.

Resultados: A partir de las narrativas de las participantes del estudio, fueron ilustrados los resultados del cuidado de mujeres en situación de violencia conyugal, y se organizaron en dos categorías representativas: Propiciar el afrontamiento a la violencia conyugal a partir del fortalecimiento de la mujer y Comprometer el afrontamiento a la violencia conyugal por causa del no cuidado, y esta última se dividió en dos subcategorías tituladas: Vulnerar a la mujer para que permanezca en la relación abusiva y Susceptibilidad de la mujer para que se enferme/muera.

Conclusión: Ofrecer una asistencia calificada en los servicios impacta directamente en la vida de las mujeres que sufren violencia, ya sea de forma positiva o negativa, dado que el no cuidado puede repercutir en el agravamiento de la salud de la mujer y puede, inclusive, provocar su muerte; mientras que, al proporcionar una atención acogedora, el profesional favorece el empoderamiento femenino y, de esta forma, contribuye con el afrontamiento a la violencia conyugal.

\section{Introdução}

A violência conjugal se configura como um persistente problema de saúde pública atrelado à cultura machista hegemônica. Diante da magnitude e da complexidade dessa problemática, esforços têm sido empenhados, nos planos internacional e nacional, no sentido de promover estratégias de cuidado com a mulher no âmbito social e institucional, para que ela consiga mecanismos para enfrentar o agravo.

Em todo o mundo, estima-se que uma em cada três mulheres vivencie a violência conjugal. ${ }^{(1)}$ Esse fenômeno representa um problema de saúde pública que se faz presente no cotidiano feminino desde a antiguidade, estando ancorado na supremacia masculina. O próprio código penal de 1890 permitia a absolvição de crimes de homicídio cometidos contra a mulher considerados passionais, sob a justificativa da legítima defesa em nome da honra masculina. ${ }^{(2)}$ Tal prerrogativa permanece, inclusive sendo utilizada na contemporaneidade como tentativa de reversão da pena em casos de traição do homem. ${ }^{(3)}$

Por se tratar de um fenômeno global, seu enfrentamento, por meio da promoção da igualdade de gênero e da autonomia feminina, foi considerado um dos objetivos prioritários do milênio, sendo este um compromisso firmado pelos governos de 191 países-membros da Organização das Nações Unidas (ONU), incluindo o Brasil.(4) Esse país também ocupa posição de destaque no cenário internacional no que se refere ao enfrentamento da violência contra a mulher, visto que foi por meio da Convenção de Belém do Pará que estabeleceu-se no continente sul-americano o direito de as mulheres viverem uma vida livre de violência, considerando esta uma violação aos direitos humanos. ${ }^{(5)}$ Foi justamente ancorada nessa convenção que foi promulgada, em 2006, a Lei Maria da Penha, a qual cria mecanismos para coibir a violência doméstica e familiar contra a mulher no país. Segundo a ONU, esta é uma das leis mais efetivas do mundo no combate à violência doméstica e familiar, perdendo apenas para países como Espanha e Chile. ${ }^{(6)}$

É importante salientar que a saída da mulher da situação de violência não se limita ao amparo jurídico-policial, sendo imprescindível a presença de uma rede de apoio institucional e social, composta por outros serviços, como saúde e ação social, além da família, dos amigos, dos vizinhos e da igreja, em uma complementaridade entre suporte social informal e formal, apoiado por agentes profissionais e agências especializadas. Esse tipo de apoio tem sido realizado de uma maneira peculiar no atual contexto de afastamento social decorrente da pandemia causada pelo novo coronavírus no país, pois estimula-se que a mulher utilize códigos, como, por exemplo, uma marcação com um " $X$ " na palma da mão, os quais sinalizem a condição de violência ao contatar amigos e familiares, assim como vizinhos, e que estes se atentem a possíveis casos de agressóes domiciliares e 
outras formas de violência, como o isolamento e a restrição de contatos, para além do razoável e ligado à proteção de contágio. ${ }^{(7)}$ Por meio desse suporte, a mulher pode se sentir fortalecida e encorajada a romper o relacionamento abusivo. Ressalta-se, contudo, que esse processo de tomada de decisão é singular, envolvendo tempo e limiares diferentes para cada pessoa - daí a relevância de um cuidado multidisciplinar permanente e persistente. ${ }^{(8)}$

Especificamente sobre a atenção em saúde, os profissionais que atuam na Atenção Primária, por estarem mais próximos da população e na principal porta de entrada dos serviços de saúde, possuem papel fundamental, tanto para a identificação das situaçóes de violência, quanto para a condução dos casos, fornecendo direcionamentos sobre os serviços disponíveis na rede que podem auxiliar as vítimas $^{(8)}$ e, ainda, para a sensibilização de outras pessoas, como vizinhos, para a importância de se envolverem na proteção mútua. Destaca-se ainda a relevância das açôes preventivas e de promoção da cultura de paz, as quais podem contribuir para que as mulheres analisem os possíveis caminhos para resolução pacífica dos conflitos e enfrentamento da violência. ${ }^{(6)}$

Sob essa perspectiva, acredita-se que, ao tempo em que práticas de cuidado adequadas podem auxiliar as mulheres a saírem da situação de violência e amenizarem os danos decorrentes desse agravo, a omissão, por sua vez, pode impactar negativamente no enfrentamento dessa problemática. Assim, questiona-se: Quais as percepçôes dos profissionais de saúde sobre os desfechos do cuidado com a mulher em situação de violência conjugal?

No intuito de responder a essa indagação, adota-se como objetivo deste estudo revelar a percepçóes dos profissionais de saúde sobre os desfechos do cuidado com a mulher em situaçáo de violência conjugal.

\section{Métodos}

Neste estudo, adotou-se a abordagem qualitativa embasada pelo referencial metodológico da Teoria Fundamentada nos Dados. Tal método compreen- de uma determinada realidade por meio dos significados a ela atribuídos, de forma que, a partir da análise sistemática dos dados encontrados, torna-se possível a criação de uma teoria. ${ }^{(9)}$

$\mathrm{O}$ estudo vincula-se a um projeto matriz intitulado "Enfrentamento da violência conjugal no âmbito do Sistema Único de Saúde: tecnologia social envolvendo mulheres, homens e profissionais da atenção básica”, que foi contemplado pelo edital Programa Pesquisa para o SUS: Gestão Compartilhada em Saúde e recebeu apoio financeiro da Fundação de Amparo à Pesquisa do Estado da Bahia em conjunto com a Secretaria da Saúde do Estado da Bahia e o Ministério da Saúde.

O lócus escolhido para o estudo foram 22 Unidades de Saúde da Família do distrito sanitário de uma capital brasileira com altas taxas de violência e vulnerabilidade social. Nelas, foram selecionados 31 profissionais de nível superior escolhidos intencionalmente, sendo 26 da Unidades de Saúde da Família e cinco do Núcleo de Apoio a Saúde da Família, representando, respectivamente, o primeiro e segundo grupo amostral. O critério de inclusão adotado foi a atuação mínima de 6 meses na Unidade de Saúde da Família; e os de exclusão, o afastamento por motivo de saúde ou a ausência nas entrevistas por três vezes sem justificativa.

As entrevistas, que ocorreram entre fevereiro e dezembro de 2019 e tiveram duração média de 50 minutos, foram orientadas por um formulário semiestruturado contendo perguntas objetivas, destinadas à caracterização dos participantes, e subjetivas, guiadas pela proposição: "Fale-me sobre os impactos relacionados ao cuidado ofertado à mulher em situação de violência conjugal". Simultaneamente, ocorreram a transcrição, a organização e a análise dos dados com auxílio do software NVivo 10, das quais emergiu a seguinte hipótese: "Os profissionais da equipe de referência da Estratégia Saúde da Família compartilham do entendimento de que psicólogas e assistentes sociais estão melhor preparados para conduzir o cuidado com a mulher em situação de violência conjugal", o que direcionou para o segundo amostral.

Conforme proposto pela Teoria Fundamentada nos Dados, os dados obtidos foram submetidos 
aos processos de codificação aberta, axial e seletiva. Daí, foram elaboradas categorias analíticas e foi formulado o fenômeno do estudo, resultante da integração dos três elementos do modelo paradigmático (condiçóes, açóes-interaçóes e consequências/resultados), que foi nomeado "Viabilizando o empoderamento da mulher em situação de violência conjugal”. Salienta-se que o objetivo do estudo se atém às consequências desse fenômeno, que emergiram dos relatos dos participantes a respeito do cuidado prestado à mulher em situação de violência conjugal no âmbito da Estratégia Saúde da Família.

A pesquisa possui aprovação do Comitê de Ética em Pesquisa e atende às recomendaçóes contidas na resolução 466/2012 do Conselho Nacional de Saúde, tendo sido aprovada por meio do parecer 2.639224. Para tanto, foram assegurados a privacidade e o anonimato das participantes, ao tempo em que as entrevistas foram realizadas individualmente, e as falas foram apresentadas em códigos, como o exemplo: "E1G1", sendo que o primeiro numeral representa a ordem da entrevista (E) e o segundo, o grupo amostral (G). Os dados, bem como os Termos de Consentimento Livre e Esclarecido assinados pelas participantes, devem permanecer arquivados por 5 anos, podendo, após esse período, serem excluídos/destruídos.

\section{Resultados}

O estudo contou com a colaboração de 31 profissionais que atuavam, em média, por 9 anos, na Unidade de Saúde da Família de um distrito sanitário localizado em uma capital do Nordeste brasileiro. Desses, 29 eram do sexo feminino e dois do sexo masculino, sendo 17 enfermeiros, cinco médicos, quatro dentistas, três psicólogos e duas assistentes sociais. A idade média foi de 38,5 anos, e $80 \%$ realizaram pós-graduação lato sensu com abrangência temática na Saúde da Família ou Saúde Pública As narrativas remeteram para duas categorias representativas, respectivamente, do cuidado ofertado ou não ofertado às mulheres no cenário da Estratégia Saúde da Família.

\section{Propiciando o enfrentamento da violência conjugal a partir do fortalecimento da mulher}

Conforme evidenciado nas falas das profissionais, o cuidado com a mulher em situaçáo de violência conjugal favoreceu o empoderamento feminino. Nesse sentido, o profissional preparado para prestar o cuidado propiciou o fortalecimento da mulher, que se sentiu apoiada e segura para romper com a relação abusiva.

Quando oriento uma mulher nessa situação percebo que ela sente que não está sozinha, que será amparada e fica fortalecida. Isso ajuda ela a enfrentar a violência. (E18 G1)

Vou mapeando com ela as possibilidades para não sofrer mais violência. Tento resgatar o suporte familiar. Eu acho que isso ajuda a mulher a se fortalecer. Ela vai escolhendo os caminhos até se sentir segura para romper [...]. Sabe que nós estamos sempre aqui de apoio para ela. (E5 G2)

\section{Comprometendo o enfrentamento da violência conjugal em razão do não cuidado}

$\mathrm{O}$ não cuidado com a mulher em situação de violência conjugal comprometeu o empoderamento feminino para o enfrentamento do agravo. A inexistência de uma gestão organizada para a identificação e a atuação diante da problemática resultou em danos, expressos a partir das seguintes subcategorias.

\section{Vulnerabilizando a mulher para permanência na relação abusiva}

As falas revelam que o náo cuidado, atrelado ao sentimento de impotência ou despreparo para lidar com o agravo, prejudicou a saída da mulher da relação de violência conjugal. Ao comprometer a implementação de ações de enfrentamento, tal contexto acabou por suscetibilizar a população feminina para a permanência na relação abusiva.

Eu não fui capacitada, [...] me sinto impotente para lidar com esses casos. Sei que isso contribui para elas permanecem em vulnerabilidade para uma nova situação de violência. (E3 G1) 
Alguns colegas não abordam, mesmo quando suspeitam. Isso é manter a pessoa no problema.(E2 G2)

\section{Suscetibilizando a mulher para 0 adoecimento/ morte}

Os profissionais reconheceram que o não cuidado com a mulher que vivenciou a violência resultou na exposição a recorrentes agressôes do parceiro. Dessa forma, a abstençáo do cuidado pôde ser um fator importante para desfechos desfavoráveis, tornando-a suscetível ao adoecimento psíquico, podendo, inclusive, levá-la ao suicídio.

Alguns profissionais não abordam violência porque acreditam que não é competência deles. Isso prejudica a mulher que segue exposta a todo tipo de agressäo. [...] continua com medo, vergonha, depressão, podendo até cometer suicídio. (E20 G1)

A mulher que sofre violência e não é cuidada adoece ainda mais, podendo levar ao óbito da vítima. (E4 G2)

\section{Discussão}

$\mathrm{O}$ estudo limita-se por não ter avaliado se o cuidado dispensado às mulheres vem sendo uma práxis orientada pela gestão, situação que pode ter influenciado nos diferentes desfechos apresentados. Apesar disso, a investigação evidencia a importância do processo de atenção às mulheres em situação de violência conjugal e oferece elementos para o direcionamento de uma gestáo no cenário da Estratégia Saúde da Família para a prevenção e o enfrentamento da violência contra a mulher, relevando a importância da formação e do treinamento dedicado ao apoio a mulheres em situaçáo de violência na conjugalidade.

As narrativas das profissionais revelam que a condução do cuidado ofertado à mulher em situação de violência conjugal no âmbito da Atenção Primária à Saúde pode favorecer ou dificultar o enfrentamento do agravo. No que tange à continuidade da mulher na relação violenta, consequência da assistência limitada e não holística, o estudo sinaliza que tal desfecho se relaciona com o pouco contato dos profissionais com a temática no decorrer de sua formaçáo, seja no contexto acadêmico ou de capacitaçóes ao longo da carreira. Estudo realizado no Nordeste brasileiro revela que essa pouca familiaridade com o assunto faz com que os profissionais deixem, inclusive, de questionar as mulheres sobre a vivência de violência, mesmo diante da suspeita, ${ }^{(10)}$ o que, para o estudo, é análogo a manter a mulher refém da situação.

Neste estudo, evidenciamos que a falta de conhecimento sobre a temática faz com que os profissionais percam a oportunidade de auxiliar as mulheres no enfrentamento do agravo, contribuindo para a manutenção da relação violenta. Para superar essa limitação, é fundamental que a capacitação para atuar diante dos casos de violência conjugal seja realizada desde a graduação, por meio da inclusão da temática nos planos de ensino, e prossiga durante a vida profissional, por meio da educação continuada. ${ }^{(11)}$ Sobre esse aspecto, o governo britânico, no ano de 2016, reconheceu que a ausência de treinamento contínuo para profissionais que atuam diante de situações de violência conjugal pode contribuir para falhas na identificação do agravo. ${ }^{(12)}$ Por outro lado, investigaçáo realizada no Reino Unido com 64 profissionais de saúde mostrou que a capacitação habilita as trabalhadoras para abordarem a temática de violência em todos os contatos com a usuária, favorecendo até mesmo o reconhecimento do evento, ainda na ausência de sinais visíveis. ${ }^{(13)} \mathrm{Tal}$ situação, assim como a revelada em estudo realizado com 33 nigerianas vítimas de violência conjugal, ${ }^{(14)}$ corrobora a importância do preparo profissional para reconhecer a vivência de abuso - condição essencial para o direcionamento da atenção com fins no enfretamento do problema.

Essa forma cuidadosa de prestar assistência, assente no conhecimento e permeada pela capacitação técnica-científica, oferece segurança para conduzir as situaçôes de violência. A abrangência acerca da complexidade que envolve o fenômeno pode contribuir para que os profissionais adotem uma postura de respeito ao tempo da mulher para romper com o ciclo da violência e náo se sintam frustrados com a morosidade do desfecho e as escolhas da vítima - 
que nem sempre vão ao encontro do que é esperado pela profissional. ${ }^{(8,15)}$ A prestação de cuidado é, dessa forma, centrada na mulher e em suas expetativas, suas necessidades e seus anseios, sem se sobrepor a ela. Assim, considerando os processos interacionais que definem ou não o cuidado dispensado pelas trabalhadoras da saúde e da intervenção social, urge uma gestão atenta para a formação em serviço com fins no enfrentamento da violência conjugal. Tal premissa já vem sendo estimulada desde a implementação da Política Nacional de Enfrentamento à Violência contra as Mulheres, em 2011, que possui enquanto eixos estruturantes a prevenção, a assistência e o enfrentamento, assumindo o agravo enquanto objeto pertencente à seara da saúde, que deve se articular com os demais serviços da Rede de Atendimento com fins na garantia de direitos à mulher. ${ }^{(16)}$

Contudo, em que pese a responsabilidade institucional em disponibilizar capacitação permanente acerca de temáticas laboriosas, de difícil abordagem, como o caso de violência conjugal, isso não exime os profissionais da responsabilidade de autonomamente buscarem o aperfeiçoamento. Assim, infere-se que o reconhecimento da incapacidade para abordar a temática de violência com a mulher, conforme sinalizam os resultados, pode ter efeito positivo se, somado a isso, for implementada uma busca para aprimorar a assistência. Isso porque, diante desse diagnóstico, planos de aprimoramento e formação individual podem ser elaborados com fins de qualificação profissional, o que contribuiria para, diante da adversidade, incrementar as habilidades para atuar e não se abster de fazer. A própria portaria 2.436 , de 21 de setembro de 2017, preconiza que os profissionais de saúde devem assumir a responsabilidade por sua capacitação e desenvolver estratégias para a qualificação de sua força de trabalho em parceria com a gestão. ${ }^{(17)}$

Vale pontuar que os profissionais podem ser responsabilizados, nas esferas civil e penal, por danos resultantes da má assistência. Tal situação, definida como imperícia, está contemplada nos códigos de éticas de diferentes profissóes da saúde, a exemplo das categorias médica e de enfermagem. ${ }^{(18,19)}$ Com isso, mais do que uma motivação, buscar capacitar- -se para a atuação diante de fenômenos emergentes da comunidade assistida configura-se enquanto uma obrigação legal. Tal situação é fundamental para promover uma assistência com base em conhecimento teórico-científico e reduzir, assim, os danos decorrentes de um cuidado ineficaz.

Embora também relacionado ao despreparo para lidar com a temática, emerge ainda o sentimento de impotência para atuar diante de situaçóes de violência conjugal. Essa inércia dos profissionais guarda relação com fatores profundos da construção social, na qual situações de violência são entendidas como restritas ao âmbito privado, devendo ser resolvidas apenas pelo casal ou no âmbito de instituiçóes jurídico-policiais, conforme sinaliza estudo desenvolvido em Santa Catarina. ${ }^{(8,20)}$ Assim, enquanto uma construção sociocultural, estudo nacional evidencia a maior aceitação da violência, sobretudo contra pessoas com identidade de gênero feminina. ${ }^{(21)}$ Essa crença também é compartilhada pelos profissionais de saúde, o que leva à não investigação do cotidiano conjugal, tido como espaço de intimidade do casal, comprometendo a atenção para o enfrentamento da problemática.

Outra situação desvelada pelo estudo, que tende a atrasar ainda mais o processo de ruptura da relação abusiva, diz respeito às condutas do profissional de indiferença diante da situação de violência. A gravidade dessa postura associa-se ao despreparo e à atitude de descaso diante das repercussóes da vivência do abuso para a vida e a saúde da mulher. Essa situação foi evidenciada em pesquisa sueca realizada com 30 profissionais de saúde que relataram a indiferença relacionada ao sentimento de insegurança individual com a temática. ${ }^{(15)}$ Essa conduta prejudica diretamente a saúde das assistidas, sendo fundamental o envolvimento da gestão, no sentido de ampliar as perspectivas de atuação e promover mudanças comportamentais junto ao corpo de trabalho.

Diante do exposto, os achados revelam a interface entre o náo cuidado e a permanência da mulher no relacionamento, evento que as vulnerabiliza para novas situaçôes de violência. Corroborando esse fato, estudo realizado com 15 profissionais de saúde na Paraíba evidencia que, quando o profissional não intervém diante dessa circunstância, favorece a per- 
petuação da violência. ${ }^{(22)} \mathrm{A}$ mesma situação foi relatada em pesquisas desenvolvidas com profissionais de saúde na África do Sul e nos Estados Unidos, as quais referiram que o desconhecimento sobre a forma de atuar limita a assistência e contribui para a permanência da mulher na relação, consequentemente a expondo a novas situaçóes violentas ${ }^{(23,24)}$ situação também desvelada em nosso estudo.

Associado à permanência na relação abusiva, o estudo alerta que, diante do náo cuidado, as mulheres estão suscetíveis ao adoecimento físico e mental, inclusive tentativas de suicídio. Nesse sentido, a Organização Pan-Americana de Saúde destaca que as mulheres inseridas em um contexto de violência por parceiro íntimo estão quase duas vezes mais propensas a problemas com álcool, depressão, tentativa de suicídio e suicídio. ${ }^{(1)}$ Ratificando essas informaçôes, estudo realizado com 11 mulheres palestinas aponta que a vivência desse agravo promove desesperança em suas vidas, fazendo com que elas considerem cercear a própria vida. ${ }^{(25)}$

Embora não seja um achado do estudo, é importante pontuar que, para além do adoecimento e do risco de suicídio, as mulheres que não contam com apoio ficam mais suscetíveis também ao feminicídio. Tal situação pode ser evidenciada por estudo divulgado em 2019 que mostra aumento nas taxas de mortalidade por feminicídio com o passar dos anos. (26) Outro estudo apontou que circunstâncias como o distanciamento social em virtude da Covid-19 vem vulnerabilizando ainda mais a mulher para o óbito. ${ }^{(27)}$ Diante disso, destaca-se a participação do profissional de enfermagem para apoiar as mulheres para o enfrentamento dessa problemática, podendo sua prática incidir na redução dos índices de morbimortalidade por esse agravo. ${ }^{(26)}$

O estudo chama a atenção para o fato dos profissionais, ao se referirem sobre as consequências relacionadas ao cuidado, sobretudo o comprometimento para a saúde e o risco de óbito, adotarem uma posição de impessoalidade, transferindo para o outro a responsabilidade pela negligência no cuidado. Isso pode guardar relaçáo com uma tentativa subconsciente de se eximir da responsabilidade pela assistência não dispensada à mulher. Esse não olhar para si limita a reflexão sobre as práticas inadequa- das implementadas, não favorecendo o aprimoramento e a mudança na forma de atuação. ${ }^{(11)}$

As falas nos fazem perceber que essa transferência de responsabilização não acontece quando os resultados das açôes e interaçóes trazem benefícios para as mulheres. Essa situação pode ser observada quando os profissionais que atendem as usuárias em situação de violência desenvolvem sua práxis guiados por boas práticas, como o interesse pela capacitação, pela sensibilidade, pelo acolhimento e pela escuta sensível e qualificada. Nessas circunstâncias, os resultados das açôes e das interações são positivos, o que significa a mulher não se permitir viver em uma relação violenta. Estudo desenvolvido no Rio Janeiro, com 16 mulheres que frequentavam as reunióes do Centro de Referência, constatou que uma escuta qualificada, respeitosa e sem julgamento por parte dos profissionais de saúde favorece o enfrentamento do fenômeno pela mulher. ${ }^{(20)}$

As profissionais revelam que essa forma empática de atuaçáo auxilia no fortalecimento da mulher para que ela consiga encontrar mecanismos para o enfrentamento da vivência de violência. Essa conduta expressa a prática de estar com o outro, além da companhia, deixando evidente o sentimento de preocupação pela vida e segurança da usuária. Estudo realizado com cem enfermeiras em um município do Rio Grande do Sul apontou experiência exitosa em relação ao cuidado prestado às mulheres em situação de violência. Essa forma de atuar foi pautada no acolhimento, no apoio, na proteção e nas orientaçóes, ressaltando a articulação com a rede de apoio local. Tais condutas possibilitaram ainda a instrumentalização das mulheres sobre seus direitos, contribuindo para uma tomada de decisão consciente e efetiva em relação à vivência. ${ }^{(28)}$

É fazendo referência a essa forma de cuidado, resolutivo e pautado em boas práticas, que a gestão deve embasar a construção de seus planos assistenciais. Para tanto, é fundamental levar em consideração, além da singularidade apresentada por cada usuária, as articulaçóes possíveis de serem estabelecidas com os diferentes atores sociais e institucionais. Estudo nacional desenvolvido com enfermeiras na Estratégia Saúde de Família de Porto Alegre evidenciou que, entre as estratégias exitosas utilizadas para o 
enfrentamento da violência contra a mulher, encontram-se o envolvimento e a articulaçáo com a rede de atenção à mulher em situação de violência. ${ }^{(29)}$

No âmbito da Atenção Primária à Saúde, esse suporte pode ser disponibilizado a partir de uma organização de serviços, pensados em articulação com a gestão nos diferentes níveis de atenção. É importante destacar que, na área de saúde, muitas vezes, a atuação carece de competências múltiplas, que nem sempre são dominadas pelos profissionais. Nesse sentido, a violência apresenta-se como um desafio complexo para o profissional, exigindo seu aprimoramento e interlocução com outros setores, descentralizando e expandindo práticas, para que sejam identificadas estratégias assertivas, evitando que as mulheres permaneçam isoladas e mais vulneráveis ao agressor, mesmo quando acompanhadas, pois é essa a condição das mulheres que não têm suas necessidades consideradas ou são negligenciadas. ${ }^{(20)}$

\section{Conclusão}

O estudo revela que receber ou não uma assistência qualificada nos serviços impacta na vida das mulheres, seja positiva ou negativamente. $\mathrm{O}$ não cuidado pode reverberar em exposição a recorrentes agressões do parceiro com consequentes agravamentos para a saúde feminina, podendo, até mesmo, resultar em óbito. Em contrapartida, a atenção, quando pautada em acolher e fortalecer a mulher, propicia o empoderamento feminino, que contribui para o enfrentamento da violência conjugal. Desse modo, o cuidado integral revela-se essencial para o fortalecimento da mulher, no sentido de romper com o ciclo de violência conjugal e se permitir uma vida sem violência.

\section{Agradecimentos}

O presente trabalho foi realizado com apoio da Coordenação de Aperfeiçoamento de Pessoal de Nivel Superior - Brasil (CAPES) - Código de Financiamento 001 - e da Fundação de Amparo à Pesquisa do Estado da Bahia.

\section{Colaborações}

Carneiro JB, Gomes NP, Almeida LCG, Campos LM, Magalhães JRF, Lírio JGS, Virgens IR e Costa DSG contribuíram com a concepção do estudo, análise e interpretação dos dados, redação do artigo, revisão crítica relevante do conteúdo intelectual e aprovação da versão final a ser publicada.

\section{Referências}

1. Organização Pan-Americana de Saúde (OPAS). Organização Mundial da Saúde (OMS). Folha informativa - Violência contra as mulheres. Brasília (DF): OPAS; OMS; 2017 [citado 2020 Nov 25]. Disponível em: https:// www.paho.org/bra/index.php?option=com_content\&view=article\&id= 5669:folha-informativa-violencia-contra-as-mulheres\&ltemid=820

2. Brasil. Presidência da República. Casa Civil. Subchefia para Assuntos Jurídicos. Decreto $n^{0} 847$, de 11 de outubro de 1890. Promulga 0 Código Penal. Brasília (DF): Presidência da República; 1890 [citado 2020 Nov 25]. Disponível em: http://www.planalto.gov.br/ccivil_03/ decreto/1851-1899/d847.htm

3. Carvalho J. Afinal, o que é a legítima defesa da honra. JusBrasil; 2020 [citado 2020 Nov 25]. Disponível em: https://canalcienciascriminais. jusbrasil.com.br/artigos/459668535/afinal-o-que-e-a-legitimadefesa-da-honra

4. Nações Unidas Brasil. Objetivo de desenvolvimento sustentável 5. Igualdade de gênero. Brasília (DF): Nações Unidas Brasil; 2020 [citado 2020 Dez 7]. Disponível em: https://brasil.un.org/pt-br/sdgs/5

5. Rangel CE, Wenczenovicz TJ. Gênero e violência: interfaces com as políticas públicas no estado do Rio Grande do Sul e Rio de Janeiro. Rev Barbarói. 2016;47:144-61.

6. Moreira CA, Cavalcanti PR. 0 combate à violência doméstica e familiar no estado de Goiás. Raízes Direito. 2018;7(2):27-55.

7. Conselho Nacional de Justiça (CNJ). Sinal Vermelho: CNJ lança campanha de ajuda a vítimas de violência doméstica na pandemia. Brasília (DF): CNJ; 2020 [citado 2020 Nov 25]. Disponível em: https:// www.cnj.jus.br/sinal-vermelho-cnj-lanca-campanha-de-ajuda-avitimas-de-violencia-domestica-na-pandemia/

8. Pereira-Gomes N, Erdmann AL, Gomes NR, Monteiro DS, Santos RM, Couto TM. Apoio social à mulher em situação de violência conjugal. Rev Salud Pública. 2015;17(6):823-35.

9. Strauss A, Corbin J. Pesquisa qualitativa: técnicas e procedimentos para 0 desenvolvimento de teoria fundamentada. 2a ed. Porto Alegre: Artmed; 2008.

10. Arboit J, Padoin SM, Vieira LB, Paula CC, Costa MC, Cortes LF. Health care for women in situations of violence: discoordination of network professionals. Rev Esc Enferm USP. 2017;51:e03207.

11. Murillo P, Sebastián MS, Vives-Cases C, Goicolea I. [Factors associated with primary care professionals' readiness to respond to intimate partner violence in Spain]. Gac Sanit. 2018;32(5):433-8. Spanish.

12. National Institute for Health and Care Excellence (NICE). Domestic violence and abuse. United Kingdom: NICE; 2016 [cited 2020 Nov 2020]. Available from: https://www.nice.org.uk/guidance/qs116/ resources/domestic-violence-and-abuse-pdf-75545301469381 
13. Dheensa S, Halliwell G, Daw J, Jones SK, Feder G. From taboo to routine: a qualitative evaluation of a hospital-based advocacy intervention for domestic violence and abuse. BMC Health Serv Res. 2020;20(1):129.

14. Fawole OI, Balogun BO, Adejimi AA, Akinsola OJ, Wyk JM. Training medical students: victim's perceptions of selectively screening women for intimate partner violence in health care settings. BMC Med Educ. 2019;19(1):196.

15. Nilsen P, Schildmeijer K, Ericsson C, Seing I, Birken S. Implementation of change in health care in Sweden: a qualitative study of professionals' change responses. Implementation Sci. 2019;14(1):51.

16. Brasil. Presidência da República. Secretaria de Políticas para as Mulheres. Política de nacional de enfrentamento à violência contra as mulheres. Brasília (DF): Secretaria de Políticas para as Mulheres; 2011 [citado 2020 Nov 25]. Disponível em: https://www12.senado. leg.br/institucional/omv/entenda-a-violencia/pdfs/politica-nacionalde-enfrentamento-a-violencia-contra-as-mulheres

17. Brasil. Ministério da Saúde. Gabinete do Ministro. Portaria $n^{0} 2.436$, de 21 de setembro de 2017. Aprova a Política Nacional de Atenção Básica, estabelecendo a revisão de diretrizes para a organização da Atenção Básica, no âmbito do Sistema Único de Saúde (SUS). Brasília (DF): Ministério da Saúde; 2017 [citado 2020 Nov 25]. Disponível em: https://bvsms.saude.gov.br/bvs/saudelegis/gm/2017/ prt2436_22_09_2017.html

18. Conselho Federal de Enfermagem (Cofen). Resolução Cofen $n^{\circ}{ }^{\circ}$ 564/2017. Brasília (DF): Cofen; 2017 [citado 2020 Nov 25]. Disponível em: http://www.cofen.gov.br/resolucao-cofen-no-5642017_59145. html

19. Conselho Federal de Medicina (CFM). Código de Ética Médica: Resolução CFM n 2.217, de 27/9/2018, modificada pelas resoluções CFM $n^{0}$ 2.22/2018 e 2.26/2019. Brasília (DF): CFM; 2019 [citado 2020 Nov 25]. Disponível em: http://portal.cfm.org.br/images/PDF/ cem2019.pdf
20. Netto LA, Moura MA, Silva GF, Penna LH, Pereira AL. Women in situation of violence by their intimate partner: making a decision to seek a specialized violence support service. Rev Gaucha Enferm. 2015;36:135-42.

21. Garcia LP, Silva GD. Violência por parceiro íntimo: perfil dos atendimentos em serviços de urgência e emergência nas capitais dos Estados brasileiros. Cad Saúde Pública. 2018;34(4):e00062317.

22. Costa DK, Reichert LP, França JR, Collet N, Reichert AP. Concepções e práticas dos profissionais de saúde acerca da violência intrafamiliar contra crianças e adolescentes. Trab Educ Saúde. 2015;13(2):79-95.

23. Wath A. Women exposed to intimate partner violence: a Foucauldian discourse analysis of South African emergency nurses' perceptions. Afr Health Sci. 2019;19(2):1849-57.

24. Brossoie N, Roberto KA. Community professionals' response to intimate partner violence against rural older women. J Elder Abuse Negl. 2015;27:470-88.

25. Baloushah S, Mohammadi N, Taghizadeh Z, Taha AA, Farnam F. A whole life of threats: a qualitative exploration of lived experiences of palestinian women suffering from intimate partner violence. Int J Womens Health. 2019;11:547-54.

26. Rabelo DP, Santos KC, Aoyama EA. Incidência da violência contra a mulher e a lei do feminicídio. Rev Bras Interdisciplinar Saúde. 2019;1(4):71-6.

27. Vieira PR, Garcia LP, Maciel EL. Isolamento social e o aumento da violência doméstica: 0 que isso nos revela? Rev Bras Epidemiol. 2020;23:e200033.

28. Acosta DF, Gomes VL, Oliveira DC, Marques SC, Gomes GC. Care for women in domestic violence situation: representations of hospital nurses. Rev Eletr Enf. 2017;19:a21.

29. Marques SS, Riquinho DL, Santos MC, Vieira LB. Strategies for identification and coping with the violence situation by intimate partners of pregnant women. Rev Gaúcha Enferm. 2017;38(3):e67593. 